\title{
Anti-islanding and Distributed Generation System
}

\author{
Meraj Alam \\ Department of Electrical and Computer Engineering, Faculty of Engineering University of \\ Porto, Porto, Portugal (merajalam@fe.up.pt)
}

\begin{abstract}
This paper proposes an anti-islanding control for a grid connected distributed generation system. The proposed control is based on $d$-q frame. The scheme using current command reference as positive feedback is utilized to drift the PCC voltage beyond the threshold limits to detect islanding within 2 seconds as prescribed by IEEE 1547. The test system configuration and parameters for anti-islanding study is prepared on IEEE 929 standards. The effectiveness of proposed anti-islanding scheme is validated by simulation done in MATLAB platform.
\end{abstract}

Subject Headings. Renewable Energy, Distributed Generation.

Author Keywords. Anti-islanding, PCC, Distributed Generation, Voltage drift.

\section{Introduction}

Islanding is a condition in which a portion/area of electrical power system remains energized by the distributed generation (DG) like PV, wind energy, diesel etc. even in the absence of grid. Unintentional islanding is an undesirable situation in a utility system as it is responsible for power quality issues, damage to operating equipment's as well as protection devices and safety hazards to personnel involved. The risk aggravates as the penetration of distributed generation (DG) into the utility system increases. It will lead to interaction between DGs and islanding occurrence will be more often. The risk of islanding has been an obstacle to DG interconnection with the utility grid (Ye 2004).

Anti-islanding scheme are basically categorized into two types: Passive and Active antiislanding schemes. In passive anti-islanding method, islanding is detected by measurement of certain parameters like voltage, current at the PCC. These measurements are used to calculate certain indices like NDZ (Non Detection Zone) which is a measure of islanding detection. The most common passive methods are OVP/UVP and OFP/UFP. These passive schemes are cost effective, technology neutral but are not efficient (Yoo 2011).

While active scheme are based upon the concept of injecting disturbances and then analyzing the changes in the system parameters at the PCC when the grid is in disconnected mode. Active schemes are neither cost effective nor technology neutral but their NDZ is very less in comparison to passive methods. However active schemes are very effective in nature but should be used carefully in multiple DGs condition because it can lead to system instability. Therefore the idea is to destabilize the DG when it is islanded which facilitates islanding detection (Wang 2009).

This paper proposes an active anti-islanding scheme which uses current command as a disturbance when multiplied by voltage signal and voltage gain as a feedback to destabilize the distributed generation under grid disconnected mode. The voltage drift gain here is not kept constant rather a variable because when DG is at low level and grid is disconnected then ratio of disturbance impact and output level of DG will be very high and system efficiency will be lowered. Therefore the current command is used as feedback signal to vary 
gain which enables the size of the injected disturbance to be adjusted corresponding to DG output power level. MATLAB simulation was carried on to validate the proposed scheme for a constant power controlled inverter.

This paper is organized as follows: Section 2 shows the proposed DG system configuration. In section 3, anti-islanding control is presented. The simulation results are discussed in section 4. Finally conclusions are presented in section 5.

\section{System Configuration}

The simplest equivalent network of a DG connected to a grid with a RLC load is shown in Figure 1.

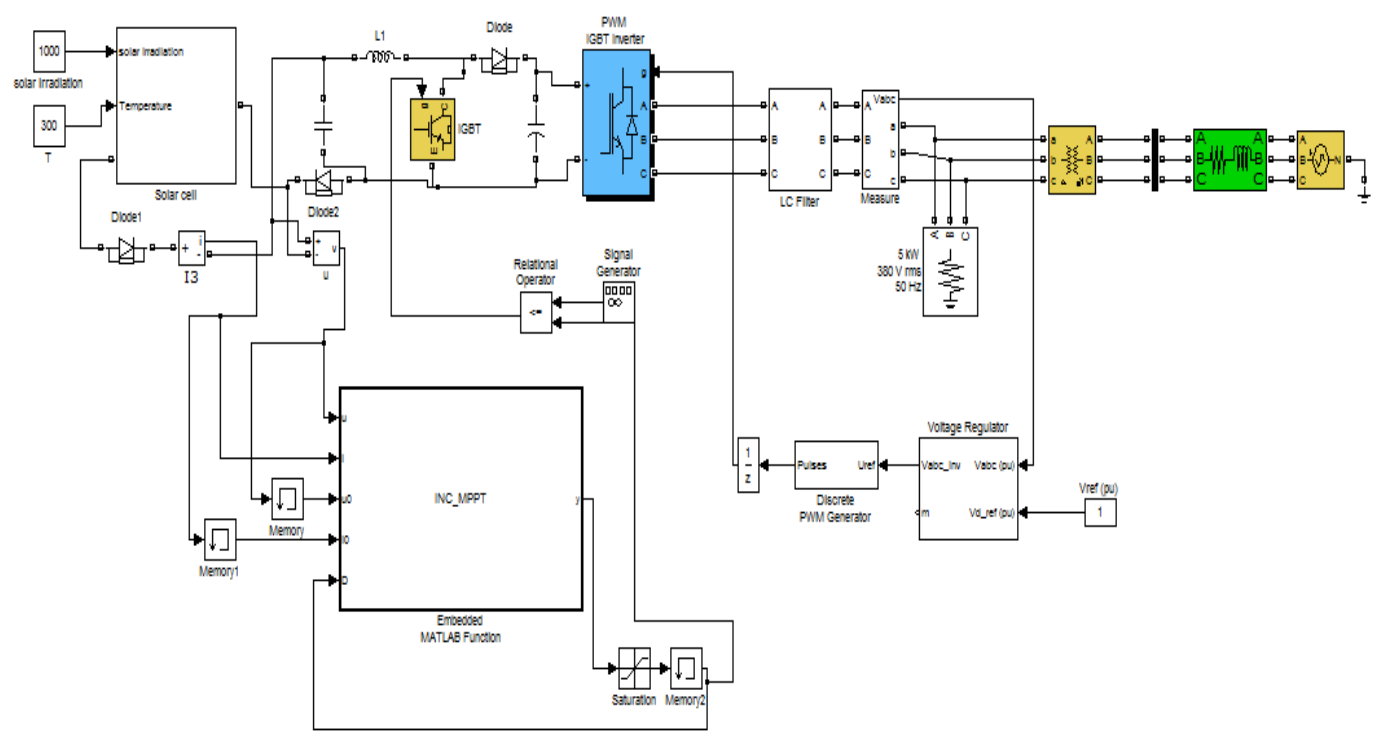

Figure 1: DG connected to grid with a RLC load in MATLAB

This network is a standard testing condition defined by IEEE 929 for typical islanding condition which would be discussed in detail in section 4. As Figure 1 suggests when the utility breaker opens due to any fault an islanding occurs.

$$
\begin{aligned}
\Delta P & =P_{\text {load }}-P_{P V} \\
\Delta Q & =Q_{\text {load }}-Q_{P V}
\end{aligned}
$$

$P_{\text {load }}$ and $Q_{\text {load }}$ are active and reactive power demand of the RLC load, respectively, and $P_{P V}$ and $Q_{P V}$ are active and reactive power generation of $D G$, respectively. If $\Delta P \neq 0, P C C$ voltage will change when islanding occurs hence OVR/UVR will detect it. Similarly, if $\Delta Q \neq 0$, the inverter control will cause the frequency of inverter output current to change until $\Delta Q=$ 0 . This can be detected by OFP/UFP [2], [9]. But if $\Delta P=0$ and $\Delta Q=0$, then it would be very difficult to detect islanding because the islanded system might be constantly operating at nominal voltage and frequency and only DG is responsible to feed the RLC load. The parameters described by IEEE for RLC load as testing condition.

- RLC load resonant frequency should be equal to grid line frequency.

- Contribution from the grid side should be zero i.e. zero power mismatches $\Delta P=0$ and $\Delta Q=0$

- The quality factor should be 1 or greater than 1.

Under this condition when breaker opens, the load and DG will resonate at nominal frequency and voltage until some mechanism which forces the voltage and frequency to go beyond the limit. 


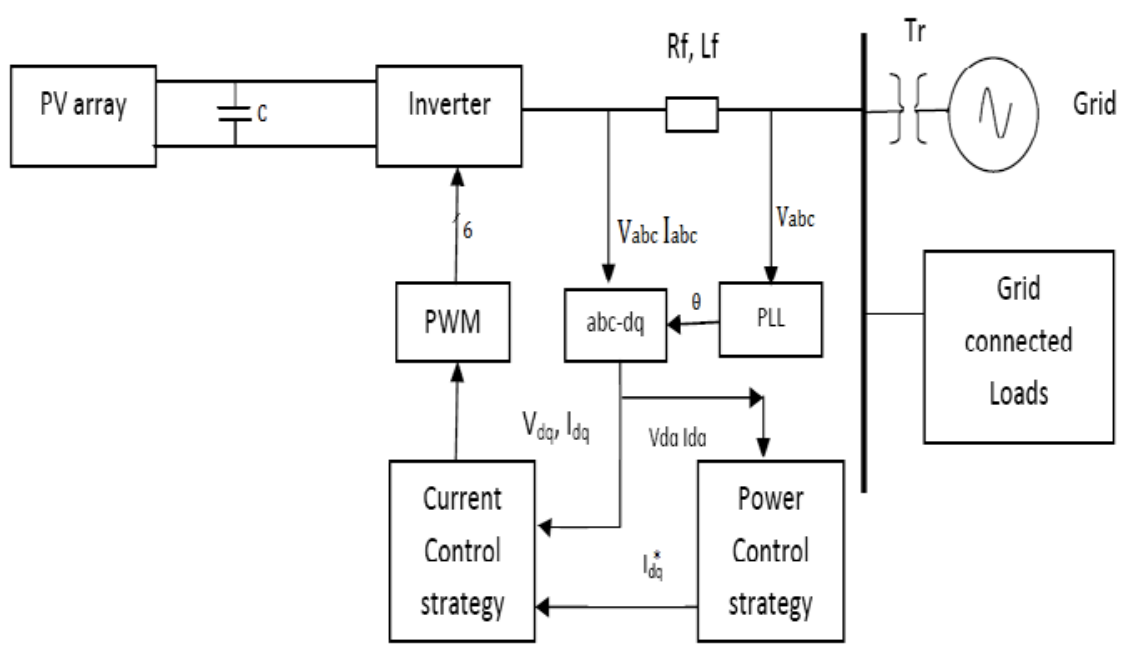

Figure 2: $d$-q control scheme of DG connected to grid

\section{Anti-islanding Control}

Figure 2 illustrates a d-q control scheme of DG connected to grid with a RLC load. Since we know that $d$-q frame is synchronously rotating reference frame, hence we assume that $d$-axis of $d$-q frame is exactly in phase with a-axis of abc frame, $V_{q}$ is zero and $V_{d}$ magnitude is equal to magnitude of inverter output voltage. The active and reactive power associated with an inverter can be explained in terms $i_{d}$ and $i_{q}$. (3) and (4) describes that active and reactive power can be regulated by $i_{d}$ and $i_{q}$ respectively (Alam 2012).

$$
\begin{gathered}
P_{i n v}=\frac{3}{2} V_{d} * i_{d} \\
Q_{i n v}=-\frac{3}{2} V_{d} * i_{q}
\end{gathered}
$$

The designed control scheme will be utilized in drifting voltage beyond threshold under islanding condition as illustrated in Figure 3 . The d-axis voltage $V_{d}$ is filtered by passing through low pass filter of cutoff frequency $100 \mathrm{~Hz}$. Hence when there is a sudden voltage change due to grid disconnection, filtered voltage will response slowly and it will create an error of voltage which is multiplied by gain and $\mathrm{d}$-axis current command reference $i_{d}{ }^{*}$ and fed as a current disturbance into the actual $i_{d 0}{ }^{*}$.

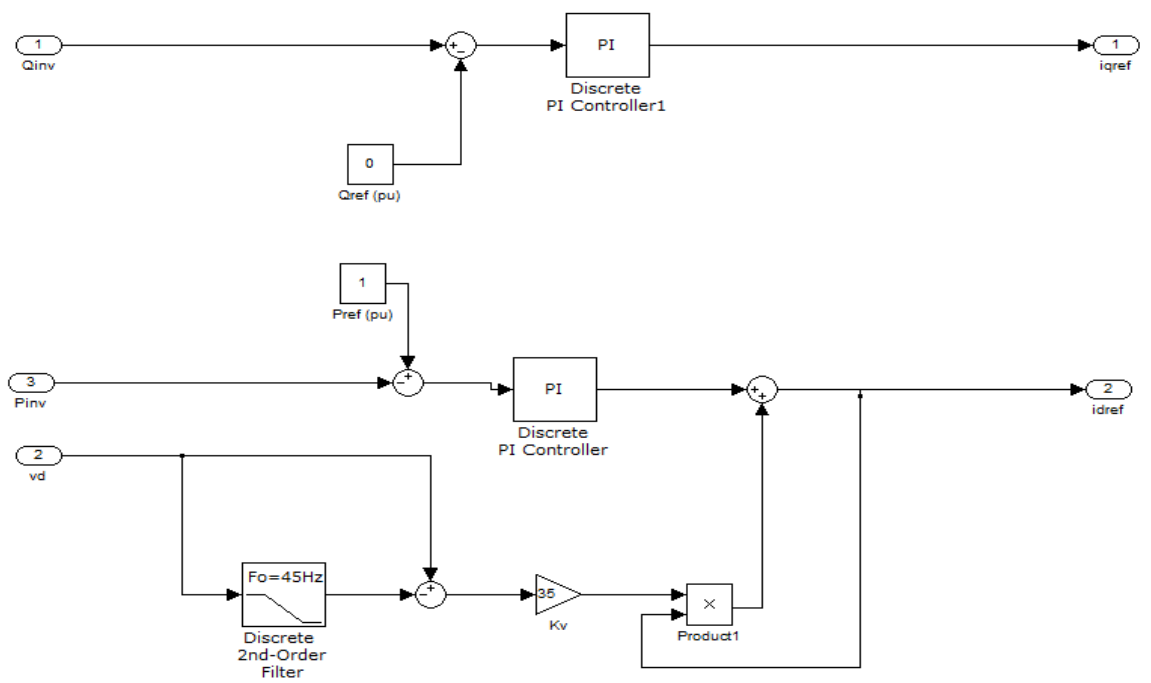

Figure 3: Power controllers with proposed current injection based voltage drift control 


\section{Simulation Results}

A DG with a constant power controlled inverter is connected to a grid of $6.6 \mathrm{kV}$ through filter, transformer and load and is represented in MATLAB model for simulation including current controller and power controller in Figure 4. The simulation parameters are mentioned in Table 1.

\begin{tabular}{ccc}
\hline Parameters & Value & Unit \\
\hline Rated Power & 3 & $\mathrm{~kW}$ \\
Nominal Voltage & 380 & Volt rms \\
Transformer ratio & $6600 / 380$ & Volt/Volt \\
Quality factor & 1 & \\
Filter frequency & 100 & $\mathrm{~Hz}$ \\
\hline
\end{tabular}

Table 1: Parameters utilized for simulation

The anti-islanding performance of RLC load with proposed scheme is presented in Figure 5 where DG and load are not matched perfectly hence load was drawing current from the grid side before the islanding occurred as shown in Figure 5(c). Islanding is easily detected at $\mathrm{Kv}=1$ within 2 seconds as voltage decreases and d-axis current rises due to grid outage and hits the threshold limits (1.1-0.9 pu).

But if the load is perfectly matched with the DG output then there is a negligible contribution from the grid as shown in Figure $6(d)$, there is no change in system voltage from $1 \mathrm{pu}$ at $\mathrm{Kv}=5$ which shows that for lower gain values islanding detection is difficult than for higher gain values, so gain should be set near upper limit. Active and reactive powers remain unaffected even after islanding as shown in Figure 6(b). Since the load is matched with the DG output, the DG continues to feed the load with minimum contribution from the grid. The reactive power requirement is zero $Q=0$ which shows the inverter is operated at unity power for maximum KWh output from the PV system.
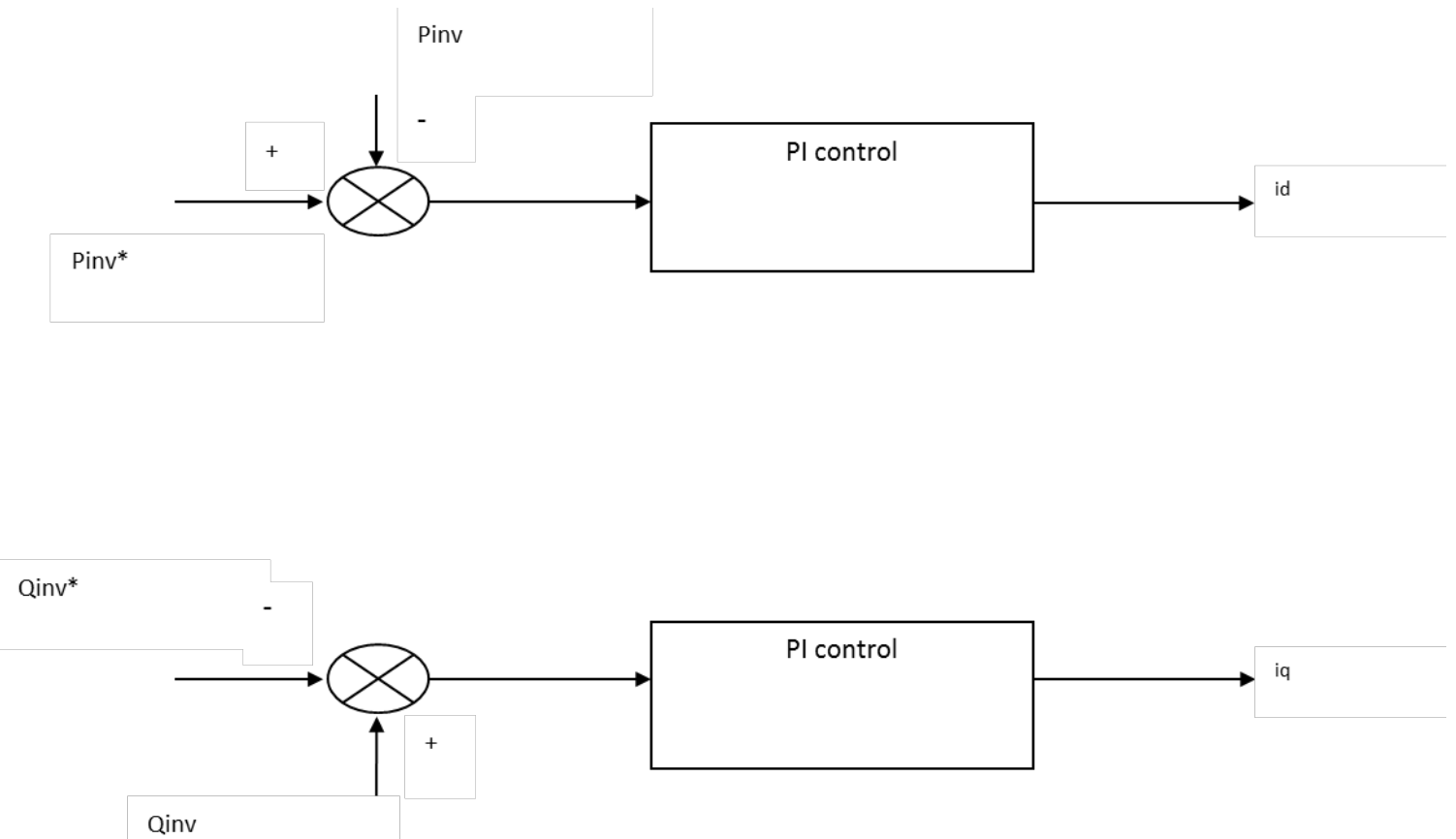

Figure 4: Power controllers 


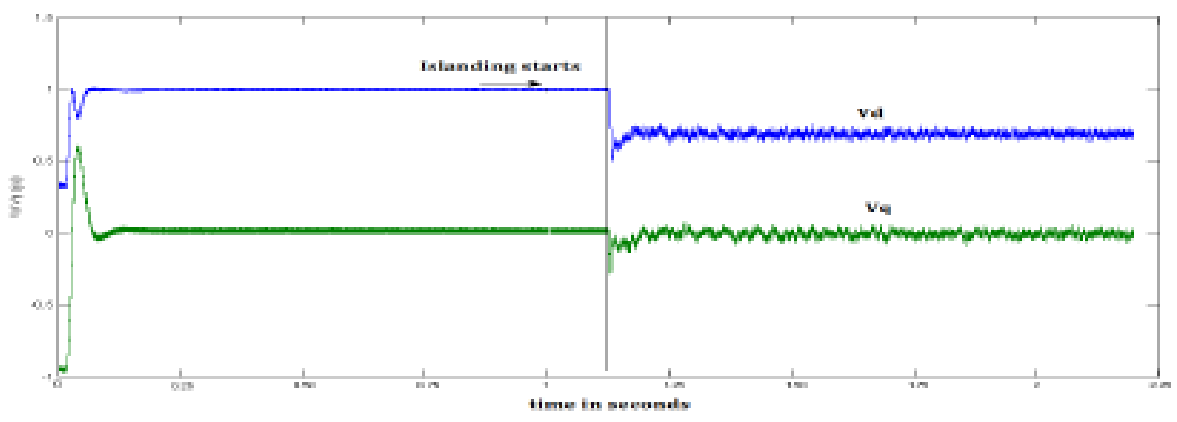

(a)

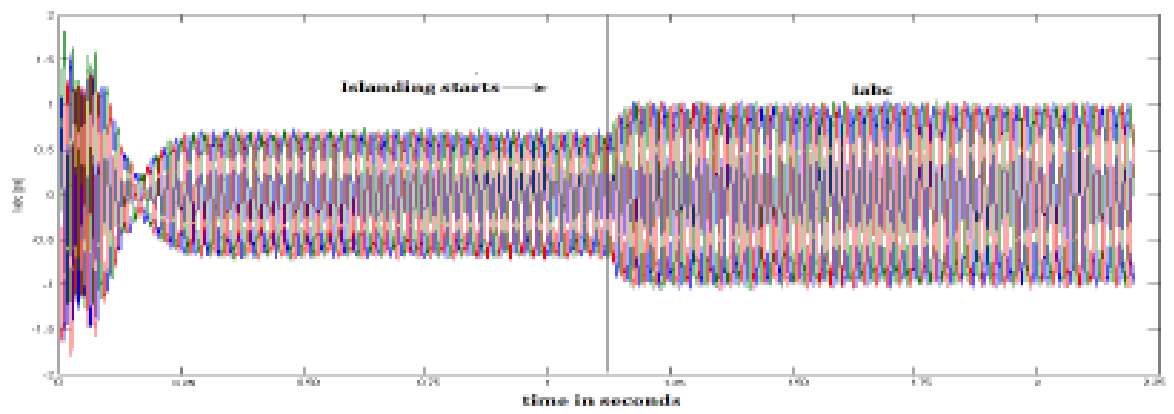

(b)

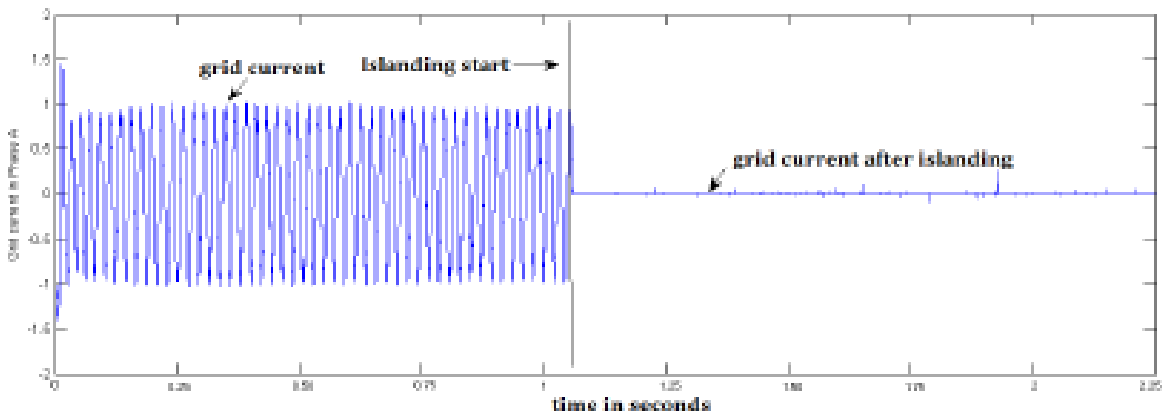

(c)

Figure 5: Anti-islanding performance of current injection based voltage drift with $\mathrm{Kv}=1$. (a) $\mathrm{V}_{\mathrm{d}}, \mathrm{V}_{\mathrm{q}}(\mathrm{b}) \mathrm{l}_{\mathrm{abc}}$ (c) Grid current

If the value of gain is set higher $\mathrm{Kv}=10$, then the controller pushes the voltage out of the limits and hence islanding is detected within 2 seconds according to IEEE standard. Hence we have to fix a gain which detects islanding phenomenon. The speed of islanding detection depends upon gain parameters and cutoff frequency for the filter. The higher the gain the more faster islanding is detected but it also causes instability in the system and for lower gain islanding will not be detected. 


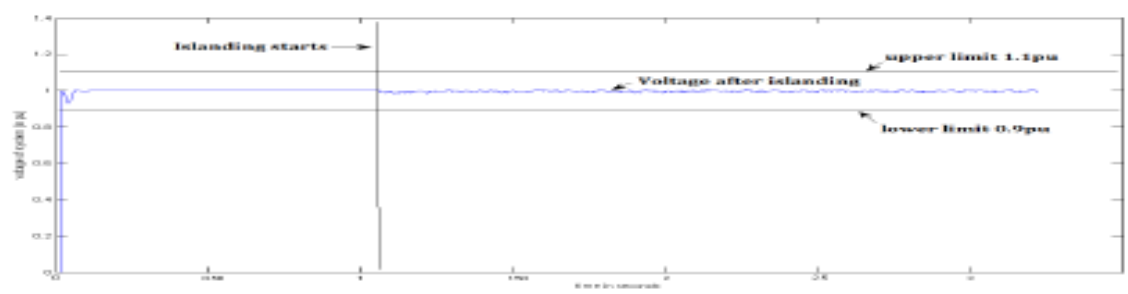

(a)

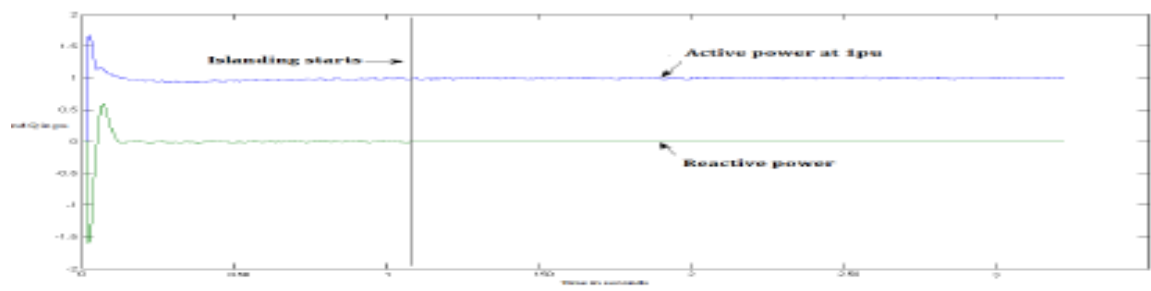

(b)

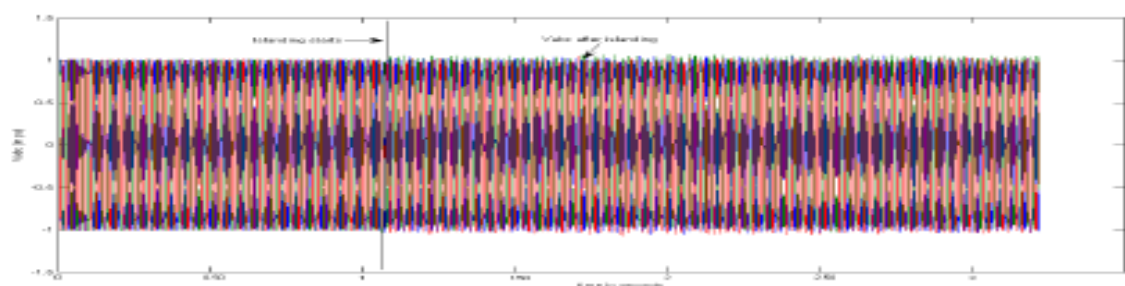

(c)

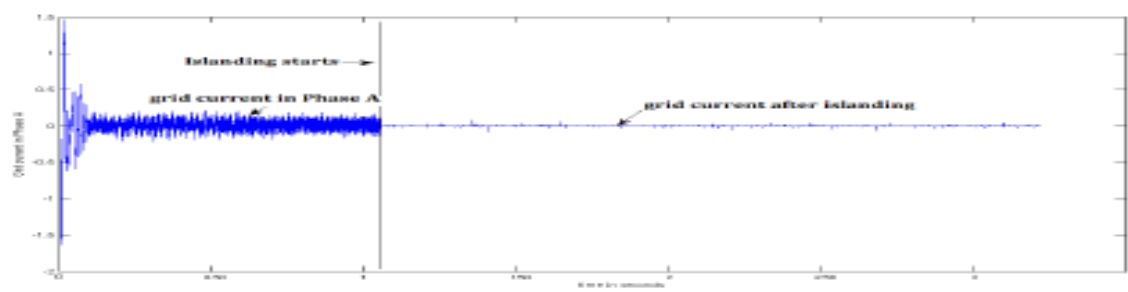

(d)

Figure 6: Anti-islanding performance of current injection based voltage drift with $\mathrm{Kv}=5$.(a) inverter voltage.(b) active and reactive power.(c) $\mathrm{V}_{\mathrm{abc} .}$ (d) Igrid

\section{Conclusions}

The voltage-drift control for the anti-islanding of DG is discussed. It aimed to eliminate influences of DG operation points on anti-islanding and output control performance of the DG, which were unavoidable in traditional schemes. Voltage drift gain had a less negative impact on the DG output control and does not depend upon quality factor and as well as integral control gain.

\section{References}

Z. Ye, A. Kolwalkar, Y. Zhang, P. Du, and R. Walling. 2004. "Evaluation of anti-islanding schemes based on nondetection zone concept". In IEEE Trans. Power Electronics 19 (5):1171-1176. 
Cheol-Hee Yoo, Du-Hee Jang, Sang-Kyoo Han, Dong-Sung Oh, and Sung-Soo Hong. 2011. "A new phase drift anti-islanding method for grid-connected inverter system". In 8th International Conference on Power Electronics - ECCE Asia.

X. Wang, W. Freitas, V. Dinavahi, and W. Xu. 2009. "Investigation of positive feedback antiislanding control for multiple inverter-based distributed generators". In IEEE Trans. Power Syst. 24 (2): 785-795.

Seul-Ki Kim, Jin-Hong Jeon, Jong-Bo Ahn, Byongjun Lee, and Sae-Hyuk Kwon. 2010. "Frequency shift acceleration control for anti-islanding of distributed generation inverters". In IEEE Transactions On Industrial Electronics 57 (2).

David Velasco, Caesar Trujillo, Gabriel Garcera, and Emilio Figueres. 2011. "An active antiislanding method based on phase-pLL perturbation". In IEEE Transactions On Power Electronics 26 (4).

M. M. Alam. 2012. "Current injection disturbance based voltage drift control anti-islanding control for a grid connected photovoltaic inverter system". In IEEE SCEECS. 